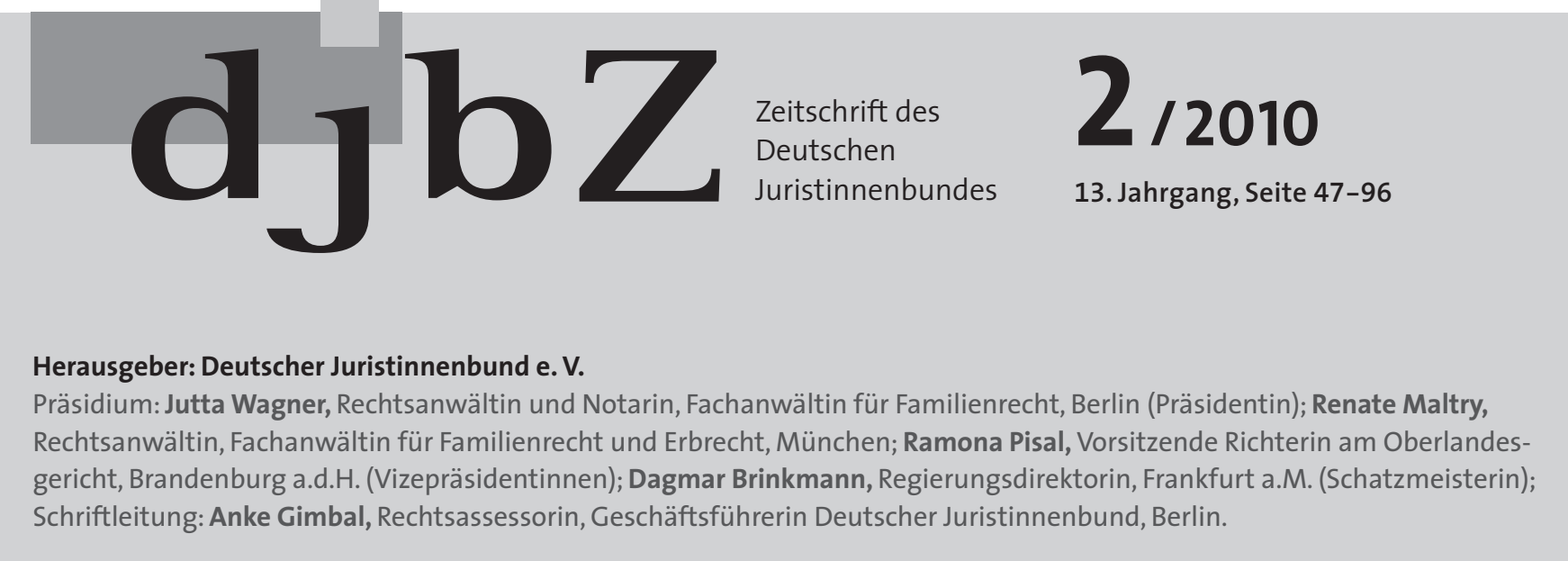

\title{
Die Gleichstellung von Frau und Mann im Erwerbsleben braucht mehr als bloße Wahlfreiheit - Bedingungen für Wahlfreiheit müssen erst geschaffen werden
}

\author{
Gabriele C. Klug \\ Mitglied der Kommission Arbeits-, Gleichstellungs- und Wirt- \\ schaftsrecht im djb, Ltd. Ministerialrätin a.D., Beigeordnete und \\ Kämmerin, Wesel
}

\section{Prof. Dr. Eva Kocher}

Mitglied der Kommission Arbeits-, Gleichstellungs- und Wirtschaftsrecht im djb, Hochschullehrerin, Europa-Universität Viadrina, Frankfurt (Oder)

\section{Prof. Dr. Sibylle Raasch}

Mitglied der Kommission Arbeits-, Gleichstellungs- und Wirtschaftsrecht im djb, Hochschullehrerin, Universität Hamburg

Das zentrale gleichstellungspolitische Ziel des Koalitionsvertrages 2009 „Wachstum. Bildung. Zusammenhalt“ für die 17. Legislaturperiode lautet: Wahlfreiheit zwischen Familienleben und Erwerbstätigkeit für Eltern, gleiche Chancen für Frauen und Männer im Beruf wie im Familienleben (S. 67). Positiv daran ist, dass neben den Frauen bei „Familie“ auch die Männer ins Blickfeld gerückt werden, Vereinbarkeit also als Problem beider Geschlechter definiert wird. Doch die hierfür angebotenen Instrumente bleiben vage oder unzureichend, die Mängel des Allgemeinen Gleichbehandlungsgesetzes (AGG) insbesondere werden nirgends thematisiert. Die Position der Frauen im Erwerbsbereich und ihr Schutz vor Diskriminierung sollen nach dem Koalitionsvertrag nicht durch verbindliche Strategien oder Instrumente grundlegend verbessert werden, sondern auf dem Wege der öffentlichen Appelle und freiwilligen Unternehmensaktivitäten. Mancherorts droht der bereits erreichten Gleichstellung der Geschlech- ter durch den Koalitionsvertrag sogar wieder Aufweichung und Verschlechterung.

Das bloße Beschwören einer „Kultur der Vielfalt“ und die Propagierung von „Diversity-Strategien“ (S. 73) allein wird für Frauen noch keine gleichberechtigte Teilhabe an der Erwerbsarbeit bringen. Managing Diversity ${ }^{1}$ ist eine vorrangig betriebswirtschaftlich ausgerichtete Strategie des Personalmanagements. Sie kann sich mit Gleichberechtigungspostulaten und Diskriminierungsverboten decken, muss es aber nicht. Denn das Ziel von Managing Diversity ist eine Optimierung des Personaleinsatzes im Betrieb. Die in einem Unternehmen bestehende Vielfalt soll nicht mehr bekämpft und eingeebnet, sondern offensiv gewinnbringend eingesetzt und vermarktet werden. Das kann bestehende Geschlechtsstereotypen und hieran anknüpfende Benachteiligungen für Frauen sogar verstetigen oder verstärken bis hin zur angeblich besonderen Eignung der Frauen für das nette Betriebsklima und das KaffeeKochen. Auch setzt die Gewinnorientierung jedem Managing Diversity enge Grenzen, indem es sich für das Unternehmen rechnen muss. Frauen müssen aber darauf bestehen, dass ihr Anspruch auf Gleichberechtigung in der Wirtschaft auch dort durchgesetzt wird, wo er dem einzelnen Unternehmen keinen Gewinn bringt, sondern es sogar etwas kostet.

Im Einzelnen benennt der Koalitionsvertrag für den Erwerbsbereich drei konkrete Handlungsfelder, auf welche im Folgenden detaillierter eingegangen werden soll.

\footnotetext{
1 Zu den möglichen Dimensionen von Managing Diversity siehe Krell, Gertraude, Diversity Management: Chancengleichheit für alle und auch als Wettbewerbsfaktor, in: dies. (Hrsg.), Chancengleichheit durch Personalpolitik, 5. Auflage, Wiesbaden 2008, 63 ff.
} 


\section{Vereinbarkeit von Beruf und Familie (S. 68 f., 73)}

Als „familienbewusste Arbeitszeit“ fordert der Koalitionsvertrag familienfreundliche und flexible Arbeitszeitmodelle und Auszeiten vom Beruf für die Familie. Auszeiten bleiben aus Frauensicht jedoch prinzipiell problematisch. Denn Frauen müssen sie mit Verlusten an Einkommen und Aufstieg selbst bezahlen, auch wenn mit dem Elterngeld inzwischen ein, nach oben allerdings begrenzter, Einkommensersatz geleistet wird. Eine Studie des Hamburger Welt WirtschaftsInstituts (HWWI) ${ }^{2}$ zeigt, dass gerade höher qualifizierte Frauen die durch eine längere Babypause eingetretenen gravierenden Verluste an Einkommen und Aufstieg im Vergleich zu nicht pausierenden Frauen zumindest bis zu ihrem 45. Lebensjahr nicht wieder einholen konnten. Auch der Gender Pay Gap, die Entgeltdifferenz zwischen Frauen und Männern, der in Deutschland im EU-Vergleich skandalöse 24 Prozent ausmacht, dürfte zu einem Teil durch die familienbedingten Berufsunterbrechungen der Frauen verursacht sein. Mütter müssen im Interesse einer geschlechtergerechten Teilhabe an Einkommen und Alterssicherung also möglichst im Beruf bleiben und sich die dennoch notwendigen Auszeiten paritätisch mit den Vätern teilen.

Vor diesem Hintergrund ist es zu begrüßen, wenn der Koalitionsvertrag die Partnermonate stärken und ein Teilelterngeld bis zu 28 Monaten einführen will. Die heutige Rechtslage nach $\mathbb{4} 4$ Abs. 2 BEEG bestraft eine paritätische Beteiligung beider Elternteile an der Kinderbetreuung via doppelter Teilzeitarbeit damit, dass sich die Bezugszeit für Elterngeld halbiert, weil der Anspruch doppelt verbraucht wird.

Bei der 2008 neu eingeführten Pflegezeit wurde das noch im Referentenentwurf enthaltene Pflegeunterstützungsgeld allerdings wieder herausgestrichen, so dass Entgeltansprüche bislang nur bei Unterbrechungen von kurzer Dauer nach \ 616 BGB bestehen. Die aktuell diskutierte Lösung, dass Beschäftigte sich eine längere Pflegezeit vorher und nachher selber erarbeiten können sollen, geht an der Pflegerealität vorbei und würde zudem praktisch erneut einseitig die Frauen mit einem Verzicht auf Erwerbsarbeitszeit und -einkommen belasten. Hier müssten ähnliche Lösungen wie bei der Elternzeit angestrebt werden. Weil sich allerdings die Pflege älterer Menschen nicht einfach in einem Jahr Auszeit ableisten lässt, wäre Pflegeteilzeit wichtig.

Das Aktionsprogramm „Perspektive Wiedereinstieg“ könnte zwar mehr Frauen eine Berufsrückkehr nach einer längeren Auszeit erleichtern, wenn es wie im Koalitionsvertrag vorgesehen ausgebaut würde. Vorrangig käme es aber darauf an, dass Menschen mit Familie gar nicht erst durch die Umstände gezwungen würden, aus der Erwerbsarbeit auszusteigen. Deshalb müssten sich die Arbeitszeiten den Bedarfen aus dem familiären Bereich anpassen, wie das der Koalitionsvertrag zwar fordert, aber nirgends konkretisiert. Bloße Kampagnen, wie im Koalitionsvertrag angekündigt, helfen jedenfalls gegenüber ökonomischen Zeitkalkülen der Unternehmen nicht weiter.

Zwar setzen inzwischen viele Unternehmen auf eine Flexibilisierung der Arbeitszeiten über variable Arbeitszeiten, Kor- ridormodelle und Arbeitszeitkonten. Die Ausdehnung, Verringerung und Positionierung von Arbeitszeit folgt dabei bislang jedoch betrieblichen, nicht aber familiären Bedarfen: So verlängerten sich die Arbeitszeiten angesichts der guten konjunkturellen Lage bis 2008 und reduzierten sich in der Krise 2009, wobei letzteres auch einen Beschäftigungsschutz barg. ${ }^{3}$ Familiäre Zeitbedarfe wird die betriebliche Arbeitszeitflexibilisierung auch künftig nur dann berücksichtigen, wenn Gesetze oder Tarifverträge dieses von den Betrieben verlangen und die Beschäftigten selber insoweit durchsetzbare Rechte erhalten. Das Teilzeit- und Befristungsgesetz (TzBfG) 2000 war hier ein Einstieg zur Verringerung der Arbeitszeit aus persönlichen Gründen, ist allerdings einen Anspruch auf erneute Arbeitszeitverlängerung bislang schuldig geblieben. Eigene Rechte der Beschäftigten, ihre regelmäßige Arbeitszeit auch ohne Reduktion entsprechend den eigenen Bedürfnissen variabel zu positionieren und eventuell sogar zeitweise von zuhause aus zu arbeiten, bietet die Arbeitszeitgesetzgebung bisher nicht. Erst die Durchsetzung von optionaler Arbeitszeit würde die Arbeitszeit nicht nur flexibel, sondern auch familienfreundlicher machen. Hierzu jedoch schweigt der Koalitionsvertrag.

\section{Verwirklichung von Entgeltgleichheit}

\section{Prekäre Beschäftigung ab- anstatt ausbauen (S. $21 \mathrm{f}$.)}

Altersarmut ist vor allem ein Problem weiblicher Lebensläufe - und dies ist in erster Linie eine Konsequenz der Tatsache, dass sich typisch weibliche Lebensläufe durch Diskontinuitäten, Beschäftigung im Niedriglohnsektor und im Vergleich mit Männern schlechtere Bezahlung auszeichnen. Die diskontinuierliche und niedrig bezahlte Beschäftigung ist durch den bislang mangelhaften sozialen Schutz in aller Regel auch prekär und führt dazu, dass die Erwerbsarbeit kaum den Lebensunterhalt zu sichern vermag, geschweige denn eine ausreichende Alterssicherung gewährleistet.

Der Koalitionsvertrag weist zwar darauf hin, dass Rente „kein Almosen“ sein und jede(r) einen Anspruch auf eine gute Rente haben sollte, die (der) ihr (sein) Leben lang hart gearbeitet hat. Allerdings schlägt er als Maßnahme hierfür nur vor, dass Ältere noch länger als bereits bisher hart arbeiten sollen: Die Koalition will „die Voraussetzungen für eine längere Teilhabe Älterer am Erwerbsleben verbessern“.

Auf der anderen Seite scheinen die mit der Prekarität verbundenen Probleme nicht gesehen zu werden: Durch Erhöhung und Dynamisierung der Grenze für sozialversicherungspflichtige Mini-Jobs (die geprüft werden soll) und eine weitere Lockerung des Befristungsverbots sollen prekäre Beschäftigungsmöglichkeiten sogar noch weiter ausgebaut werden. Künftig soll eine sachgrundlose Befristung beim selben Arbeitgeber nach einem Jahr Wartezeit erneut möglich sein. Damit

2 Vgl. Boll, Christina, Lohneinbußen durch geburtsbedingte Erwerbsunterbrechungen, HWWI Research Paper 1-19, Hamburg 2009.

3 Vgl. Herzog-Stein, Alexander/Seifert, Hartmut, Deutsches „Beschäftigungswunder" und flexible Arbeitszeiten, WSI-Diskussionspapier Nr. 169, Düsseldorf Februar 2010. 
würden Umgehungen des Verbots unzulässiger Befristungsketten ermöglicht.

\section{Gesetzlicher Mindestlohn (S. 21)}

Eine zentrale Rolle bei der Gewährleistung existenzsichernder Erwerbsarbeit haben Mindestlohnregelungen. Sie könnten ein wichtiges Instrument der Existenzsicherung und Alterssicherung gerade von Frauen sein, die in deutlich höherem Ausmaß als Männer im Niedriglohnsektor arbeiten ${ }^{4}$. Nach dem Koalitionsvertrag soll nun zur „Verhinderung von Lohndumping“ (also nicht zur Gewährleistung existenzsichernder Erwerbsarbeit) „die Rechtsprechung zum Verbot sittenwidriger Löhne [...] gesetzlich festgeschrieben werden “. Dies wäre eine bloße Kodifizierung und Festschreibung des Status Quo der Rechtsprechung. Anders als die Koalition meint, kann damit gerade nicht „wirksam gegen soziale Verwerfungen in einzelnen Branchen" vorgegangen werden.

Der Koalitionsvertrag lehnt aber einen „einheitlichen gesetzlichen Mindestlohn“ nicht nur explizit ab, er stellt sogar die bisherigen Ansätze in Richtung Mindestentgelt infrage: Sie sollen bis Oktober 2011 evaluiert werden. Das Ergebnis dieser Evaluierung, in der nur Beschäftigungswirkungen eine Rolle spielen sollen, nicht aber die soziale Integration, die Existenzsicherung von Vollzeitbeschäftigten oder die Belastung der Sozialsysteme mit Aufstock-Leistungen, soll als Grundlage für die Entscheidung dienen, ob die geltenden Mindestlohnregelungen Bestand haben oder aufgehoben werden sollten.

Der damit verbundene Hinweis auf die Tarifautonomie, die Vorrang vor staatlicher Lohnfestsetzung habe, führt in die Irre. Denn tarifautonome Entgeltfestsetzung kann nur dort die sozialen Funktionen eines Mindestentgelts erfüllen, wo es ausreichende tarifliche Repräsentativität gibt - dies ist in vielen Bereichen, in denen typischerweise Frauen arbeiten, jedoch gerade nicht der Fall. Und die Mindestentgeltfestsetzung in Anlehnung an Tarifverträge (Allgemeinverbindlicherklärung) soll sogar deutlich erschwert werden: Wenn der Tarifausschuss in dem die konkreten Tarifparteien gar nicht Mitglied sind wie vorgesehen gestärkt wird, werden die Tarifparteien geschwächt. Wenn die Allgemeinverbindlicherklärung auf dem Verordnungswege nur noch nach Einvernehmen im Kabinett sowie im Tarifausschuss möglich sein soll, wie es der Koalitionsvertrag vorsieht, wird dieses Instrument praktisch wohl nicht mehr angewandt werden können.

\section{Gleichstellung und Bekämpfung der Entgeltdiskriminierung (S. 73/74)}

Die Entgeltungleichheit zwischen den Geschlechtern ist mittlerweile als Thema im öffentlichen Bewusstsein - schließlich liegt die Entgeltungleichheit zwischen den Geschlechtern in Deutschland weit über dem europäischen Durchschnitt und hat sich in den letzten Jahrzehnten kaum verbessert ${ }^{5}$. Die Koalitionäre haben sich insofern zu Recht das Ziel gesetzt, die Entgeltungleichheit zu überwinden und das Prinzip „gleicher Lohn für gleiche Arbeit“ für Frauen und Männer umzusetzen. Vergessen wird dabei jedoch, dass das geltende Recht viel wei- ter geht: Es bietet auch bei gleichwertiger Arbeit von Frauen und Männern den Anspruch auf gleiches Entgelt. Dies zu verwirklichen ist unionsrechtliche Pflicht, nicht nationale Kür. Die Koalitionsvereinbarung nennt auch die Instrumente, die sich die Koalition zur Verwirklichung der Entgeltgleichheit vorstellt: So soll zunächst in der Wirtschaft dafür geworben werden, das beratungsunterstützte Lohntestverfahren LogibD („Lohngleichheitsinstrument des Bundes - Deutschland“) einzusetzen. Mit diesem Selbsttestinstrument wird auf Analysen reagiert, nach denen wesentliche Gründe für die Entgeltungleichheit in den betrieblichen Entgeltstrukturen zu suchen $\operatorname{sind}^{6}$.

Bei genauer Betrachtung zeigt sich jedoch, dass dieses Instrument keine brauchbaren Informationen liefert. Es ist damit nicht nur ein untaugliches Mittel zur Bekämpfung von Entgeltdiskriminierung, sondern konterkariert das vorgegebene Ziel sogar. Denn die Kriterien, die Logib-D für „objektive“ Erklärungen von Entgeltdifferenzen hält - vor allem die Kriterien „, berufliche Stellung im Betrieb“ und „Anforderungsniveau des Arbeitsplatzes“ - enthalten häufig selbst Diskriminierungen. Logib-D klammert insbesondere die geschlechtsdiskriminierende Bewertung von Tätigkeiten aus, die eine der Hauptursachen für Entgeltdiskriminierung darstellt. Statt Unternehmen zu einer geschlechtergerechten Bewertung von Tätigkeiten zu ermutigen, täuscht der Selbsttest über bestehende Entgeltdiskriminierung hinweg und dürfte viele Unternehmen trügerisch in Sicherheit wiegen, indem er bestehende Entgeltungleichheiten verschleiert. ${ }^{7}$ Diese Befürchtungen haben sich übrigens bereits realisiert: So hat der Geschäftsführer des Kosmetikkonzerns Weleda, Erik Schuchardt, mit Hilfe von LogibD lediglich herausgefunden, dass im Unternehmen „nur“ eine (im Vergleich zu 24 Prozent unterdurchschnittliche) Entgeltdifferenz von acht Prozent und deshalb kein Handlungsbedarf bestehe ${ }^{8}$.

Ohnehin dürften singuläre Maßnahmen, die nicht das gesamte Akteursfeld der Entgeltdiskriminierung ansprechen, kaum Erfolg versprechen. Wie wenig abgestimmt die Maßnahmen der Regierung sind, ist nicht zuletzt daran zu erkennen, dass die Vereinbarung zwischen der Bundesregierung und den Spitzenverbänden der deutschen Wirtschaft ${ }^{9}$ keinerlei Vorschläge für Maßnahmen zur Herstellung von Entgelt-

4 Vgl. Bosch, Gerhard/Weinkopf, Claudia, Mindestlöhne - eine Strategie gegen Lohn- und Sozialdumping? in: Friedrich-Ebert-Stiftung (Hrsg.), Sozialer Ausgleich in den alten und neuen Mitgliedstaaten der EU, 2006, 26 ff.; Bosch, Gerhard/Weinkopf, Claudia, Gesetzliche Mindestlöhne auch in Deutschland?, Bonn 2006.

5 Z.B. KOM(2008)10 endg. 13; KOM(2007)424, 21; KOM(2009)694; working staff document (SEC(2009)1706).

6 BT-Drucks. 14/8952.

7 Vgl. im Einzelnen dazu die Stellungnahme des djb vom 9.3.2010 (Homepage djb).

8 SPIEGEL vom 3.2.2010 („Familienministerin Köhler schafft per Mausklick Gerechtigkeit").

9 Vereinbarung zwischen der Bundesregierung und den Spitzenverbänden der deutschen Wirtschaft zur Förderung der Chancengleichheit von Frauen und Männern in der Privatwirtschaft (2. Juli 2001), <http://www.dihk.de/inhalt/download/ chancengleichheit.pdf> (Zugriff 20.4.2010). 
gleichheit enthält, sondern selbst entgegen dem aktuellen Ansatz mit Logib-D immer noch davon ausgeht, die geschlechtsbezogenen Einkommensunterschiede könnten allein durch eine deutliche Erhöhung des Beschäftigungsanteils von Frauen verringert werden.

Ob die Koalition künftig überhaupt noch auf diese freiwillige Vereinbarung setzt, lässt sie allerdings im Vagen: Die „Bundesinitiative zur Gleichstellung von Frauen in der Wirtschaft“ soll künftig in einen „Rahmenplan zur gleichberechtigten Teilhabe von Frauen und Männern in allen Phasen des Lebensverlaufs" einbezogen werden. Unwahrscheinlich ist, dass damit am Ende der Legislaturperiode - entsprechend der Koalitionsvereinbarung - tatsächlich „[gemeinsame] Anstrengungen zur Überwindung der Entgeltungleichheit“ bilanziert werden können. Falls diese Formulierung so zu verstehen ist, dass die Koalition sich für die gesellschaftliche Aufgabe der Überwindung der Entgeltungleichheit auf freiwilliges Handeln der Unternehmen verlassen will, hat sich ihr Konzept bisher als erfolglos erwiesen, wie die jahrzehntelange Stagnation in Sachen Entgeltgleichheit, aber auch bei Frauen in Führungspositionen zeigt.

\section{Mehr Frauen in Führungspositionen und Aufsichtsräten}

\section{(S. 23, 74)}

Der Anteil von Frauen in Führungspositionen soll nach dem Koalitionsvertrag zwar maßgeblich erhöht werden. Das ist angesichts der krassen Unterrepräsentanz von Frauen in den Spitzengremien großer Unternehmen ${ }^{10}$ zu begrüßen. Dennoch sieht die Koalition keine Frauenquoten für den Corporate Governance Kodex vor. Stattdessen soll ein Stufenplan zur Erhöhung des Anteils von Frauen in Vorständen und Aufsichtsräten vorgelegt werden, dessen Einzelheiten - vor allem zeitliche Dimensionen - offen bleiben. In der ersten Stufe sind für die Unternehmen verbindlich nur Berichtspflichten geplant sowie eine transparente Selbstverpflichtung.

Einen eindrucksvollen Aufschluss über die strukturellen Verhältnisse über alle Sektoren gibt die im März 2010 vom BMFSJ herausgegebene Sinus-Studie ${ }^{11}$ : Beträgt der Anteil von Frauen an der Gesamtbevölkerung 51 Prozent und spiegelt sich diese Relation bei den Hochschulabsolvent(inn)en noch wider, so ändern sich die Verhältnisse beim beruflichen Aufstieg drastisch: Frauen sind in Führungspositionen allgemein mit 31 Prozent repräsentiert, auf der Vorstandsebene mit drei Prozent und in den Aufsichtsräten mit elf Prozent. Nach wie vor sind 75 Prozent der weiblichen Aufsichtsratsmitglieder im Rahmen von Mitbestimmungsregelungen von den Arbeitnehmervertretungen entsandt. ${ }^{12}$

Auf Frauen in Führungspositionen und den Spitzengremien kommt es für eine nachhaltige Wirtschaftsaufstellung jedoch dringend an: Mehr Frauen in Führungspositionen sind ein Schlüssel zu wirtschaftlicher Stabilität und Wachstum in der EU, stellte zuletzt ein im März 2010 vorgelegter Bericht der EU-Kommission fest ${ }^{13}$. Nach den Ergebnissen einer finnischen Studie ${ }^{14}$ sind Unternehmen mit einem geschlechterparitätisch besetzten Board im Durchschnitt um 10 Prozent profitabler als solche mit einem nur mit Männern besetzten Führungsgremium. Frauenpräsenz in den Spitzengremien und im Spitzenmanagement des Unternehmens trägt also positiv zu Betriebsergebnis, Wettbewerbsfähigkeit und nachhaltiger Aufstellung des Unternehmens bei.

Diese Erkenntnisse haben inzwischen sogar einzelne große Unternehmen - jüngst in der Bundesrepublik die Telekom zur Verabschiedung unternehmensbezogener Zielquoten veranlasst. Auch der Deutsche Corporate Governance Kodex erst 2009 war eine Empfehlung zur Berücksichtigung von Diversity bei der Zusammensetzung der Gremien eingeführt worden - soll nun erweitert werden: Die Aufsichtsräte börsennotierter Unternehmen sollen künftig konkrete Planungen und Zeitpläne für eine angemessene Vertretung von Frauen im Aufsichtsrat darlegen und über die Umsetzung in der Entsprechenserklärung nach $\mathbb{\$} 161 \mathrm{AktG}$ berichten. ${ }^{15}$ Die Einführung einer Quote bei der Besetzung von Spitzengremien bleibt in den Verlautbarungen allerdings weiterhin unerwähnt. Von den DAX-Unternehmen sollen Berichten zufolge ${ }^{16}$ lediglich EON und Daimler bereit sein, dem Beispiel der Telekom zu folgen.

Vor diesem Hintergrund stellt sich auch in der Bundesrepublik die Frage nach einer gesetzlichen (Ziel-)Quote. Die Verfestigung der lang andauernden und strukturellen Benachteiligung von Frauen in den Spitzengremien der Wirtschaft und im Spitzenmanagement stellt eine rechtswidrige Diskriminierung dar, die der Staat nicht länger tatenlos hinnehmen darf. Das Grundgesetz formuliert mit Art. 3 Abs. 2 Satz 2 GG auch Schutzpflichten im Sinne eines Untermaßverbots.

Verschiedene europäische Staaten haben bereits gesetzliche Frauenquoten für Aufsichtsräte mit hervorragenden Ergebnissen eingeführt: Norwegen kennt seit 2008 die verbindliche Quote von 40 Prozent weiblicher Aufsichtsratsmitglieder in den großen Unternehmen. Spanien hat 2007 eine 30 ProzentQuote beschlossen. In Frankreich und den Niederlanden sind kürzlich gesetzliche Regelungen zur Einführung von (Ziel-) Quoten auf den Weg gebracht oder bereits verabschiedet worden: In Frankreich soll in Geschäftsführungen und Aufsichtsräten börsennotierter Unternehmen innerhalb der kommenden sechs Jahre eine Quote von mindestens 40 Prozent angestrebt werden - das wäre eine deutliche Erhöhung gegenüber dem Anteil von 10 Prozent im Jahr 2009. In den Niederlanden sollen Unternehmen mit mindestens 250 Mitarbeitern 30 Prozent der Geschäftsführungen und Aufsichtsratspositionen mit

$10 \mathrm{Vgl}$. Holst, Elke/Wiemer, Anita, Frauen in Spitzengremien großer Unternehmen weiterhin massiv unterrepräsentiert, DIW Wochenbericht Nr. 4/2010, 2 ff.

11 Vgl. Sinus Sociovision-Wippermann: Barrieren und Brücken Frauen in Führungspositionen, BMFSFJ (Hrsg.), Berlin März 2010.

12 Vgl. Holst/Wiemer 2010, a.a.O.

13 Vgl. EU-Kommission: More Women in Senior Positions - Key to Economic Stability and Growth, Brüssel, März 2010.

14 Vgl. Kotiranta, Annu/Kovalainen, Anne/Rouvinen, Petri, Female Leadership and Firm Profitability, Finnish Business and Policy Forum, EVA analysis Nr. 3, 24.9.2007.

15 Vgl. Regierungskommission Deutscher Corporate Governance Kodex, Geschäftsstelle, Information zum 2. FidAR-Forum, Berlin, 15.3.2010.

16 Vgl. Financial Times Deutschland vom 16.3.2010, 2. 
Frauen besetzen - eine Verdoppelung des Anteils von 15 Prozent im Jahr 2009.

Gesetzliche Quoten sind ein zulässiges und wirksames Instrument zur Beseitigung der Unterrepräsentanz von Frauen in den Spitzengremien der Wirtschaft. Frost und Linnainmaa haben schon 2007 ausgeführt, dass solche Quoten für Aufsichtsräte mit dem Gemeinschaftsrecht und dem Grundgesetz vereinbar wären. ${ }^{17}$ Raasch hat 2009 diese Position vertieft und zudem gezeigt, dass die Quoten in Norwegen inzwischen nicht nur von den Unternehmen im Staatsbesitz, sondern auch von den meisten privaten Unternehmen eingelöst werden konnten und darüber hinaus sogar bei Unternehmen der Frauenanteil in den Führungspositionen gestiegen ist, die gar nicht der Quote unterlagen. ${ }^{18}$ Freiwillig, auch das zeigt das Beispiel Norwegen, sind die Privaten diesen Weg allerdings nicht gegangen. Sie hätten dazu zwei Jahre lang Zeit gehabt, bevor die verbindlichen Quoten in Kraft treten sollten, blieben aber untätig.

Nichts spricht dafür, dass die deutschen Unternehmen im Gegensatz zu diesen norwegischen Erfahrungen auf breiter Front freiwillig gegen ihre gläsernen Decken vorgehen und Frauen an ihre Spitzen bringen werden. Der djb ist daher im Rahmen eines vom BMFSJ geförderten Aktionsprojektes dazu übergegangen, die Vorstände auf Aktionärsversammlungen zu fragen, was sie zur Gleichstellung der Geschlechter und insbesondere zur Verstärkung der Frauenrepräsentanz auf Führungspositionen in ihren Unternehmen im vergangenen Geschäftsjahr getan haben und künftig zu tun gedenken. Der öffentliche Druck auf Unternehmen soll dadurch verstärkt und das Problembewusstsein auf Unternehmens-, aber auch auf Aktionärsseite geschärft werden. Das allein reicht aber keinesfalls aus, um tatsächliche Veränderungen anzustoßen.

Der Staat bleibt weiterhin gefordert, nach EU-Recht und nach dem Gleichberechtigungspostulat des Grundgesetzes. Aber der Koalitionsvertrag „Wachstum. Bildung. Zusammenhalt“ zeigt nicht, dass die Koalitionäre sich dieser Aufgabe ernsthaft stellen wollen, weder bei den Arbeitszeiten noch beim Entgelt und auch nicht zur Verstärkung der Frauenrepräsentanz in Führungspositionen und Aufsichtsräten.

17 Vgl. Frost, Ina Anne/Linnainmaa, Leena, Corporate Governance: Frauen im Aufsichtsrat - Können wir von unseren skandinavischen Nachbarn lernen?, in: Die Aktiengesellschaft 17/2007, $601 \mathrm{ff}$.

18 Vgl. Raasch, Sibylle, Erhöhung der Frauenrepräsentanz in Aufsichtsräten: Norwegen - ein Modell?, in: Zeitschrift für Europarecht, Internationales Privatrecht und Rechtsvergleichung 5/2009, 216 ff.

\section{Koalitionsvertrag 2009: \\ Bausteine einer modernen, geschlechtergerechten Familien- und Generationenpolitik?'}

\section{Prof. Dr. Margarete Schuler-Harms}

Vorsitzende der Kommission Recht der sozialen Sicherung, Familienlastenausgleich des djb; Professorin für Öffentliches Recht, insbes. Öffentliches Wirtschafts- und Umweltrecht an der HelmutSchmidt-Universität/Universität der Bundeswehr in Hamburg

\section{Effektivität, Effizienz und Evaluation als Parameter einer „aktivierenden“ Politik}

Das Schiff der Familienpolitik hat im Verlauf der vergangenen drei Legislaturperioden langsam seinen Kurs geändert und zuletzt unter der großen Koalition schnellere Fahrt aufgenommen. Der Koalitionsvertrag schreibt diese Entwicklung fort. Die neue Frauen- und Familienministerin beginnt zusätzliche Akzente zu setzen, scheint den eingeschlagenen Kurs aber grundsätzlich beibehalten zu wollen.

Der Koalitionsvertrag akzentuiert die Aufgabe einer „modernen Familienpolitik für alle Generationen“. Die Steuerund Sozialpolitik stellt er unter den Auftrag größerer Kinderund Familienfreundlichkeit und günstiger Rahmenbedingungen für Familien mit dem Ziel, die Zahl der Geburten zu erhöhen. Die klare Ausrichtung auf Nachwuchssicherung er- fordert - zumal in Zeiten schmaler Kassen - eine zielgenaue Konzeption des Familienleistungsausgleichs. Die Koalitionsparteien versprechen „Wahlfreiheit bei der Gestaltung von Familien- und Erwerbstätigkeit“ und eine Orientierung der Förderung „in der Lebenswirklichkeit von Familien“.

Ein solchermaßen aktivierendes Konzept des Sozial- und Steuerstaates erfordert eine Politik aus einem Guss und damit auch Instrumente, mit denen sich die Wirkung der Maßnahmen auf die Familien beobachten und die Maßnahmen selbst weiterentwickeln lassen. ${ }^{2}$ Unter der Großen Koalition wurde sion Recht der sozialen Sicherung, Familienlastenausgleich. Ergänzend wurden die Informationen des BMFSFJ über „Vorhaben 2010“ für den Ausschuss für Familie, Senioren, Frauen und Jugend des Deutschen Bundestages vom 18.1.2010 herangezogen, abrufbar unter <http://news.einformation.de/v3/client/media/193/ data/20094.pdf> (letztmals überprüft am 12.4.2010). Für weiterführende Hinweise danke ich - stellvertretend - den Kommissionsmitgliedern Prof. Dr. Susanne Dern (Leitung der Fachgruppe Grundsicherung, Arbeitsmarktintegration), Dr. Christine Maurer (Leitung der Fachgruppe Steuerrecht, Kindergeld) und Prof. Dr. Astrid Wallrabenstein (Leitung der Fachgruppe Vorsorge).

2 Zum Konzept des ,aktivierenden Sozialstaats“ näher Kingreen, JZ 2004, 938 (946 f.); Kersten, Die Verwaltung 2007, 309 ff., insbes. 312 f. 